\title{
Repeat Pass SAR Interferometry at VHF Band
}

\author{
A.J. Wilkinson, R.T. Lord and M.R. Inggs \\ Radar Remote Sensing Group, Department of Electrical Engineering \\ University of Cape Town, Private Bag, Rondebosch 7701, South Africa \\ Email: rlord@rrsg.ee.uct.ac.za Web: http://rrsg.ee.uct.ac.za \\ Tel: +27216502792 Fax: +27216503465
}

\begin{abstract}
The South African SAR system (SASAR) has flown a number of repeat pass interferometry flights, and this paper reports on the results achieved to date with the system. The flight geometry, data acquisition and processing are described, and examples of interferograms are shown. This paper also shows simulations of fringe patterns obtained from an interferogram simulator developed in the UCT Radar Remote Sensing Group. The simulated fringes obtained from a DEM are compared to those obtained from the real SAR data.
\end{abstract}

\section{INTRODUCTION}

The results of the first interferometric experiments of the SASAR system were published in [1]. The data acquisition campaign took place over a mountainous, forested region on the coast to the west of Cape Town, from which there was significant backscatter. Interferometric fringes were obtained, but the coherence exhibited an along-track banded nature, which resulted from difficulties in registering the data owing to local variations in azimuth ground sample spacing. Furthermore, high coherence fringes were only obtained in areas of natural surfaces covered by significant vegetation, whereas bald earth areas backscatter comparatively little energy, resulting in severe loss of coherence [2]. It was concluded that the poor SNR obtained from unvegetated terrain was a severely limiting factor for interferometry in the VHF band, resulting in patchy interferograms which would be difficult to unwrap.

This paper reports on the progress to date regarding SASAR repeat pass interferometry. Significant improvements were obtained regarding the registration of the single look complex (SLC) images. Unfortunately, the resulting interferograms did not agree well with simulated fringes that were obtained from a digital elevation model (DEM) of the area. Possible reasons for this discrepancy as well as potential solutions are discussed in this paper.

\section{DATA ACQUiSITION AND SAR PROCESSING}

The data presented in this paper were acquired from three passes over the Hermanus coastline (specifically the Botrivier lagoon and mountains to the west) of South Africa (approximate latitude $34.5^{\circ}$, longitude $19.25^{\circ}$ ). The SASAR system flew aboard a Dakota DC3 (C47TP) with a time separation of approximately 25 minutes between passes. The vertical baselines were $105 \mathrm{~m}, 206 \mathrm{~m}$ and $311 \mathrm{~m}$. The swath extends from nadir out to approximately $28 \mathrm{~km}$, covering a wide range of look angles from vertical to about $82^{\circ}$. The SAR imaging geometry is depicted (to scale) in Fig. 1. A summary of the
TABLE I

TYPICAL SASAR SYSTEM PARAMETERS.

\begin{tabular}{|l|l|}
\hline Flight height & 3000 to $4000 \mathrm{~m}$ \\
\hline Flight speed & $80-90 \mathrm{~m} / \mathrm{s}$ \\
\hline Observation angle (at $h=4000 \mathrm{~m})$ & from 0 to $\sim 85 \mathrm{deg}$ \\
\hline Range (near-far) & $24 \mathrm{~km}$ \\
\hline Range Bandwidth & $12 \mathrm{MHz}$ \\
\hline Range Resolution & $12 \mathrm{~m}$ \\
\hline Processed Azimuth Beamwidth & $6 \mathrm{deg}$ \\
\hline Corresponding Azimuth Resolution & $12 \mathrm{~m}$ \\
\hline Carrier Frequency & $141 \mathrm{MHz}$ \\
\hline
\end{tabular}

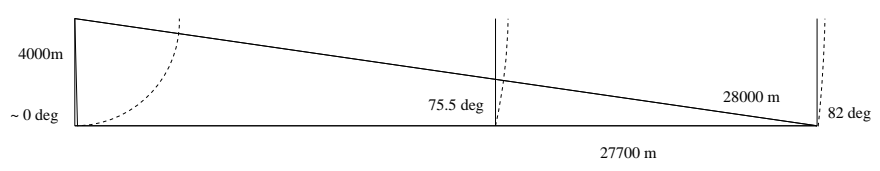

Fig. 1. Typical SASAR imaging geometry — to scale.

system parameters is given in Table I. An example of a processed image covering about half of the usable swath is shown in Fig. 3.

The raw data were processed to single look complex images using an in-house range/Doppler processor with the resolution parameters given in Table I. Prior to formation of the SAR images, each range profile was filtered using an adaptive frequency-domain notch filter to suppress radio frequency interference (RFI). Motion compensation was applied to the data to correct for deviations from a straight path. The interferometric processing was performed using modules from the GAMMA interferometric suite [3] as well as in-house developed software.

\section{IMAGE REGISTRATION}

Previous work [1] has shown that the application of a bilinear warping function is not sufficient to register the SLC images properly. After carefully correlating the two images, a surface plot can be obtained, as depicted in Fig. 2a, showing the azimuth offset required to warp image 2 onto image 1. From Fig. 2a it can be seen that the azimuth offset does not vary linearly along an azimuth line, but has a "step-like" nature. A bilinear warping function does not have sufficient "warping freedom" to follow the local variation. 

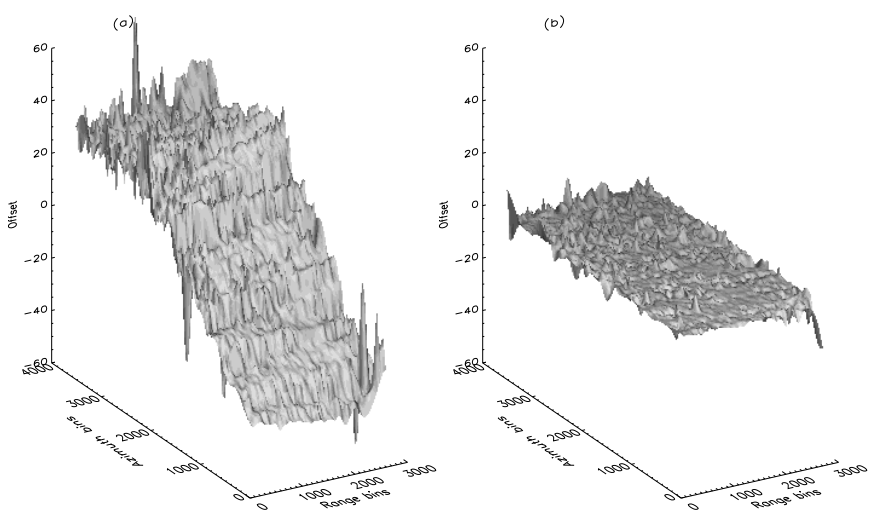

Fig. 2. Azimuth offset in samples before and after applying warping function.

A registration algorithm was developed, which is able to warp the image with an arbitrary amount of freedom by performing localised warping on smaller patches of the image. Correlating the two resulting registered images produces the plot shown in Fig. 2b, which shows that the required azimuth offset is close to zero across the image. This warping algorithm significantly improved the registration and hence the coherence between the two images.

Originally it was thought that the discrete steps in the required azimuth offset were the result of "drop-outs" occurring in the data recording system (i.e. missing range lines). However, it is now believed that these steps occur due to the platform's deviation from the nominal flight path, causing a "bunching up" of range lines as the aircraft moves away from the nominal flight path and a "stretching out" of range lines when it flies on, or parallel to, the nominal flight path. Future work will focus on correcting this irregular spacing in azimuth by interpolating each azimuth line as part of the motion compensation module.

\section{REsults}

In order to check the predicted performance, a model-based interferometric simulator [4] was employed, in order to generate noisy interferograms and coherence maps for the proposed flight geometries. An example of the simulator output is shown in Fig. 4, which depicts the noisy interferogram and the flattened interferogram of the Hermanus/Botrivier area for the case of a $100 \mathrm{~m}$ vertical baseline.

Fig. 5 shows the real interferogram and flattened interferogram of the same area that was obtained from processing the SASAR data using the $105 \mathrm{~m}$ vertical baseline. Unfortunately, there is no immediately visible agreement between the real and simulated interferograms. There are a number of reasons for this discrepancy:

- Motion compensation was performed to mid-range. Due to SASAR's large change in look angle from near to far swath, the phase error at the near and far range is significant. This phase error becomes very problematic because the deviation of the aircraft's flight path from a straight line can be as much as $100 \mathrm{~m}$ from the nominal straight flight path. A solution to this problem might be to perform the motion compensation on blocks of range data, thereby minimising the phase errors. It might also be worthwhile to write a time-domain SAR processor which circumvents these motion compensation problems. - It is suspected that the position data contains errors in the order of a few wavelengths, due to inaccurate GPS hardware and/or accelerometers on board the aircraft. It is therefore important to invest in the best possible position measuring hardware.

- It has already been mentioned that it is believed that the azimuth data is irregularly spaced. It should be possible to interpolate the azimuth data to an equally spaced grid. It would also be beneficial to slave the PRF to the ground speed (which is not the case with the current SASAR system), and to time-stamp the data.

- Non-parallel flight paths also affect the interferogram, although this problem is not as serious as the previous ones. A stable platform such as a Boeing would help to minimise motion errors.

\section{CONCLUSIONS}

Our latest attempts at extracting interferometric information from repeat pass data acquired with the SASAR system have again shown that high coherence fringes can be obtained using repeat pass interferometry at VHF band. Unfortunately, the obtained interferograms exhibit little correlation with the topography, which means the extraction of reliable height information is not feasible without improving the current system. It is believed that the most significant interferometric errors are caused by inaccurate motion compensation.

The original system design had included the ability to slave PRF to ground speed, as well as time-stamp all recorded data to a common time base. This was unfortunately omitted in the implementation, but we will make strong representations for this to be re-instated.

\section{ACKNOWLEDGEMENTS}

The authors acknowledge the significant contribution of the SANDF DRDB and CSIR in gathering the data sets. The Hermanus data collection was funded by De Beers Consolidated Mines (Pty) Ltd.

\section{REFERENCES}

[1] A.J. Wilkinson, J.M. Horrell and M.R. Inggs, "SASAR VHF Interferometry: Early Results," EUSAR 2000, Munich, Germany, May 2000, pp. 411414.

[2] J.M. Horrell and M.R. Inggs, "Semi Desert and Forest Measurements using the South African VHF SAR System," EUSAR 2000, Munich, Germany, May 2000, pp. 145-148.

[3] U. Wegmuller and C.L. Werner, "GAMMA SAR Processor and Interferometry Software," Third ERS Scientific Symposium, vol. 3, no. ESA SP414, May 1997, pp. 1687-1692.

[4] L.S. Wray, A.J. Wilkinson and M.R. Inggs, "Synthetic Aperture Radar Simulator for Interferometry," ISRSE 2000, 28-31 March 2000. 

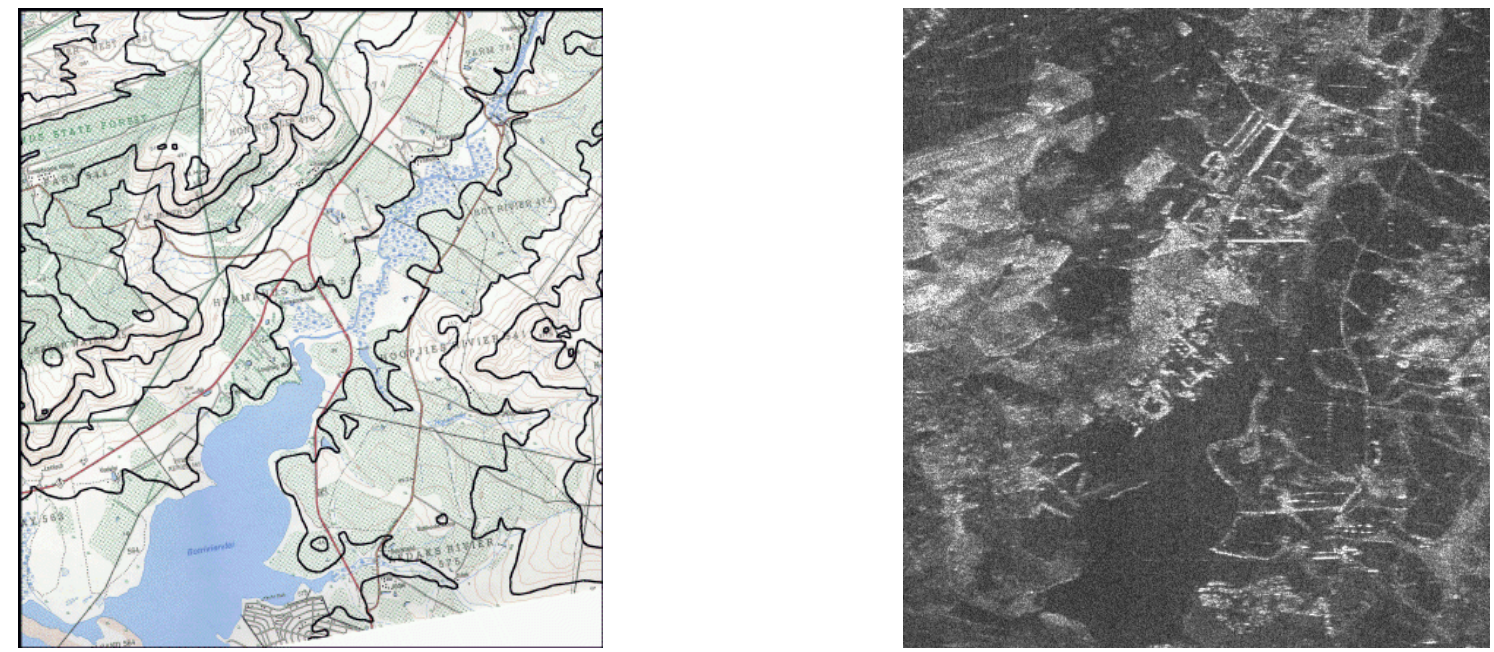

Fig. 3. Map and intensity image of Hermanus/Botrivier area of South Africa.
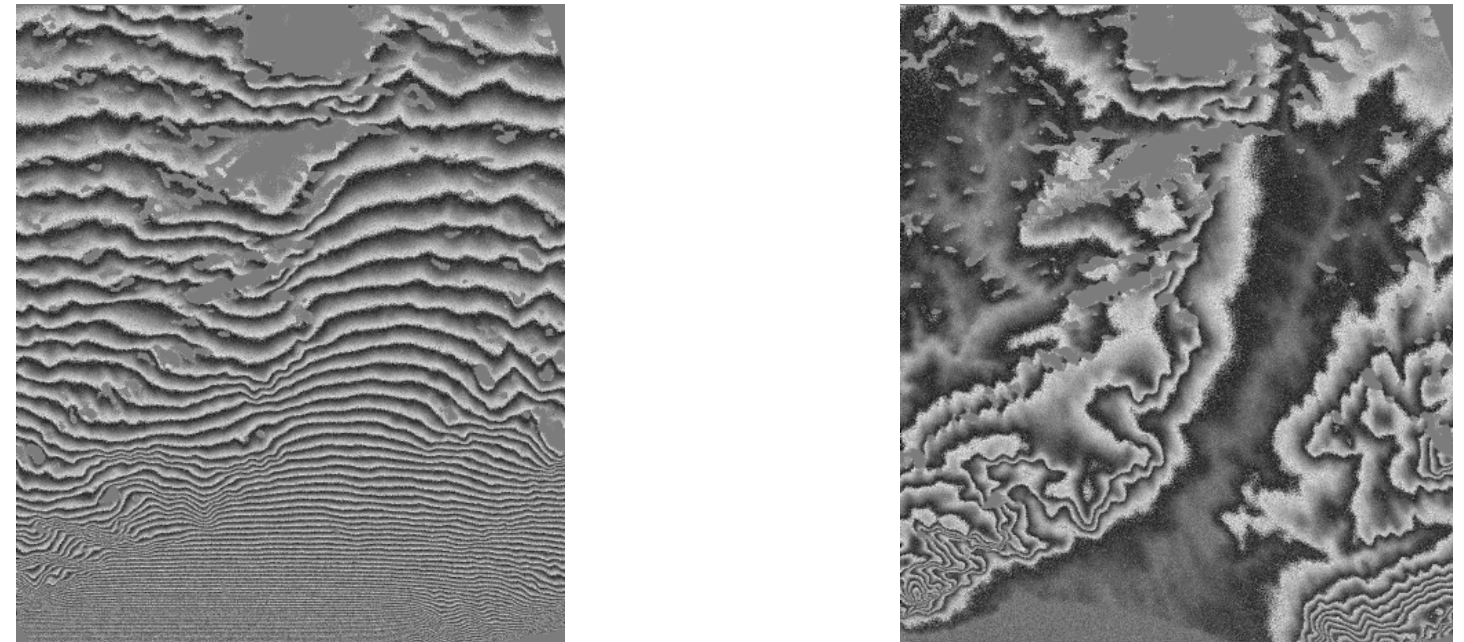

Fig. 4. Simulated interferogram and flattened simulated interferogram of Hermanus/Botrivier area of South Africa.
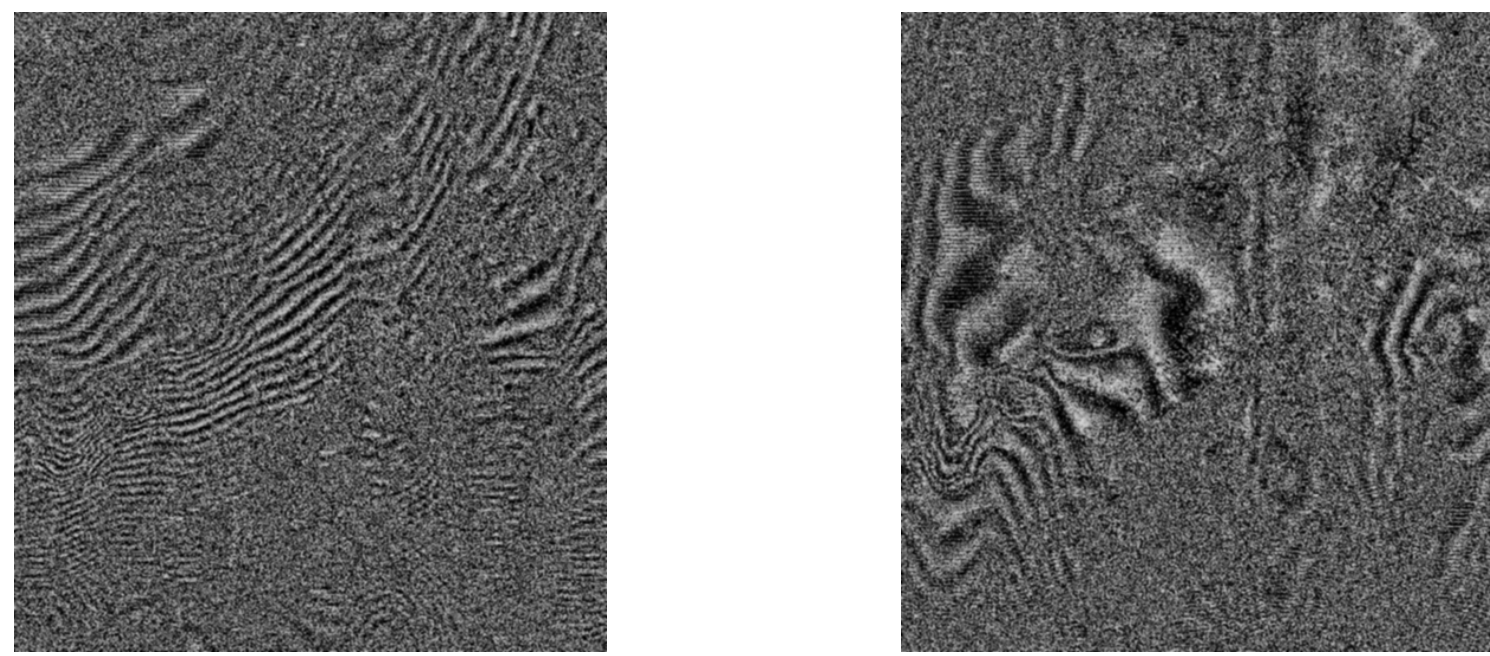

Fig. 5. Interferogram and flattened interferogram of Hermanus/Botrivier area of South Africa. 